Physical characterization of single convergent beam device for teletherapy: theoretical and Monte Carlo approach

This content has been downloaded from IOPscience. Please scroll down to see the full text. 2015 Phys. Med. Biol. 607191

(http://iopscience.iop.org/0031-9155/60/18/7191)

View the table of contents for this issue, or go to the journal homepage for more

Download details:

IP Address: 200.16.16.13

This content was downloaded on 08/09/2015 at 19:08

Please note that terms and conditions apply. 


\title{
Physical characterization of single convergent beam device for teletherapy: theoretical and Monte Carlo approach
}

\author{
R G Figueroa ${ }^{1}$ and M Valente ${ }^{2}$ \\ ${ }^{1}$ Universidad de La Frontera, Francisco Salazar 1145, Temuco, IX Región, Chile \\ ${ }^{2}$ CONICET and Universidad Nacional de Córdoba, Av. Haya de la Torre s/n, \\ Córdoba, Argentina
}

E-mail: valente@famaf.unc.edu.ar

Received 16 January 2015, revised 28 June 2015

Accepted for publication 3 July 2015

Published 7 September 2015

\section{Abstract}

The main purpose of this work is to determine the feasibility and physical characteristics of a new teletherapy device of radiation therapy based on the application of a convergent $\mathrm{x}$-ray beam of energies like those used in radiotherapy providing highly concentrated dose delivery to the target. We have denominated it Convergent Beam Radio Therapy (CBRT). Analytical methods are developed first in order to determine the dosimetry characteristic of an ideal convergent photon beam in a hypothetical water phantom. Then, using the PENELOPE Monte Carlo code, a similar convergent beam that is applied to the water phantom is compared with that of the analytical method.

The CBRT device (Converay ${ }^{\circledR}$ ) is designed to adapt to the head of LINACs. The converging beam photon effect is achieved thanks to the perpendicular impact of LINAC electrons on a large thin spherical cap target where Bremsstrahlung is generated (high-energy $\mathrm{x}$-rays). This way, the electrons impact upon various points of the cap (CBRT condition), aimed at the focal point. With the $\mathrm{X}$ radiation (Bremsstrahlung) directed forward, a system of movable collimators emits many beams from the output that make a virtually definitive convergent beam.

Other Monte Carlo simulations are performed using realistic conditions. The simulations are performed for a thin target in the shape of a large, thin, spherical cap, with an $\mathrm{r}$ radius of around $10-30 \mathrm{~cm}$ and a curvature radius of approximately 70 to $100 \mathrm{~cm}$, and a cubed water phantom centered in the focal point of the cap. All the interaction mechanisms of the Bremsstrahlung radiation with the phantom are taken into consideration for different energies and cap thicknesses.

Also, the magnitudes of the electric and/or magnetic fields, which are necessary to divert clinical-use electron beams ( 0.1 to $20 \mathrm{MeV})$, are determined 
using electromagnetism equations with relativistic corrections. This way the above-mentioned beam is manipulated and guided for its perpendicular impact upon the spherical cap.

The first results that were achieved show in-depth dose peaks, having shapes qualitatively similar to those from hadrontherapy techniques. The obtained results demonstrate that in-depth dose peaks are generated at the focus point or isocenter. These results are consistent with those obtained with Monte Carlo codes. The peak-focus is independent of the energy of the photon beam, though its intensity is not. The realistic results achieved with the Monte Carlo code show that the Bremsstrahlung generated on the thin cap is mainly directed towards the focus point. The aperture angle at each impact point depends primarily on the energy beam, the atomic number $\mathrm{Z}$ and the thickness of the target. There is also a poly-collimator coaxial to the cap or ring with many holes, permitting a clean convergent-exit x-ray beam with a dose distribution that is similar to the ideal case. The electric and magnetic fields needed to control the deflection of the electron beams in the CBRT geometry are highly feasible using specially designed electric and/or magnetic devices that, respectively, have voltage and current values that are technically achievable. However, it was found that magnetic devices represent a more suitable option for electron beam control, especially at high energies. The main conclusion is that the development of such a device is feasible. Due to its features, this technology might be considered a powerful new tool for external radiotherapy with photons.

Keywords: radiotherapy, Monte Carlo simulation, collimation, convergent beam radiotherapy $(\mathrm{CBRT})$

(Some figures may appear in colour only in the online journal)

\section{Introduction}

Current devices for external radiotherapy with linear accelerators (LINAC) act as an almost virtually punctual radiation source. This source generates a divergent radiation field of highenergy X photons, which are generated by the impact of LINAC electrons with a heavy-metal target located at the head of the accelerator, at a distance from the isocenter of about one meter. To achieve the maximum dose in the tumor it is necessary to overlap divergent fields from different angles, all of them pointing towards the isocenter. It is also necessary that each of these radiation fields is collimated and properly formed.

These features attempt to minimize radiation to healthy tissues and organs. Nowadays, with the implementation of modern technological improvements such as intensity-modulated radiotherapy (IMRT) (Spirou and Chui 1994, Phillips et al 1999, Webb 2003), this technique allows more highly concentrated radiation dose levels and high capability to conform the distribution of doses around the tumor, while dose levels to the surrounding critical structures are minimized or kept as low as possible (Phillips et al 1999, Kestin et al 2000, Das et al 2003, Bohoslavsky et al 2013). Although IMRT offers high performance, as assessed by modern treatment modalities, its implementation and daily use might have some drawbacks. Depending on the specific IMRT technique implemented, it is possible that these modalities could include prolonged treatment time and also require special immobilization devices and extra quality assurance procedures. Similar drawbacks might be applicable to other modern 
teletherapy techniques, like arc therapy with intensity modulation (Yu 1995, Otto 2008, Anderson et al 2012) and guided imaging (IGRT) (Jaffray et al 2007, Verellen et al 2007, Cui et al 2013). Even the most recently developed irradiation apparatuses, such as True Beam Varian (Maxim 2011) and Versa HD Elekta (Freeman 2013), do not use a flattening filter (FFF), which allows them to increase the dose rate by almost a factor of 3. FFFs allow much shorter treatment because their beams are divergent in nature and have many moving parts.

Some modern computer-aided technological advances like Cyber Knife (Bassalow and Rodebaugh 2006) and tomotherapy (Mackie et al 1993) have shown high performance and promising capability for improving radiation therapy quality. Cyber Knife consists of a robotic radio surgical system capable of non-invasive treatment throughout the whole body. Tumors are easily accessed with this technique thanks to its robotic structure of six degrees of freedom combined with a small $6 \mathrm{MeV}$ accelerator located at one end of its robotic arm. However, the system has drawbacks similar to those already described for devices with many movable parts.

Another newly developed technique of radiotherapy-radiosurgery is the Gyro-knife (Szeifert et al 2008, Yao-zhen et al 2013). Using a rotating quasi-parallel beam, this technique produces highly concentrated dose distributions. However, it utilizes a radioactive source of ${ }^{60} \mathrm{Co}$ incorporating more mobile parts than other known techniques, thus significantly increasing requirements for high precision on treatment positioning due to the high radiation dose applied to the target. Gamma Knife is another high-performance radiation treatment technique for high dose concentration (Meltsner and DeWerd 2008, Nie et al 2012). This method uses ${ }^{60} \mathrm{Co}$ sources, concentrating photon fluence at variably shaped targets, ranging from several millimeters up to more than 2 centimeters in diameter. It is possible to get beams focused precisely on target volumes, thereby minimizing the effects on surrounding critical structures. However, this methodology exhibits a significant limitation: currently it is applied only to intracranial treatment. Additionally both Gyro and Gamma Knife have important drawbacks: (1) the necessary regular replacement of the radioactive source and the associated disposal of radioactive waste, thus increasing operating costs; and (2) the need for new, accurate and timeconsuming quality assurance procedures.

Another radiosurgery technique conceptually similar to Gamma Knife, X-knife (Andrews et al 2006), utilizes highly collimated x-ray beams from clinical linear accelerators to achieve highly concentrated dose distributions. X-knife solves the problem of radioactive waste and reduces costs by using installed facilities. On the other hand, it requires a multi-stage scanning process by means of several irradiation arcs, which increases treatment complexity and reacquired irradiation time.

In summary, modern radiation treatment modalities allow the application of high-dose gradients, thus permitting tumor dose escalation, which increases the odds of malignant-cell elimination while protecting healthy structures. Hence, it might be suitable to consider a photon beam of inherent convergent characteristics in order to improve dose delivery techniques. This work describes the main features of a new strategy for producing a unique convergent beam for external radiotherapy without movable parts for CBRT (Convergent Beam Radiotherapy) operation. The main design characteristics and accessories of a dedicated device for CBRT and a preliminary prototype are detailed in the corresponding patent application (Figueroa and Valente 2013).

\section{Methods}

The methodology to determine the physical characteristics of the convergent beam device is as follows. We first present the CBRT approach, then analyze the analytical model for the 
distribution of doses of a single and ideal convergent beam and compare the results with those achieved with code PENELOPE Monte Carlo Simulation. We then conduct a Monte Carlo simulation of the actual situation from the impact of electrons on a cap and finally determine the magnitudes of the Electric/Magnetic field necessary to control the trajectories of electrons in the CBRT geometry.

\section{1. $C B R T$ approach}

The main idea of this proposal is to consider that the suitable manipulation of an electron beam generated as usual by clinical linear accelerators or other sources may constitute the starting point for assessing an electron current impinging normally on a relatively thin spherical shell or cap anode. Once this goal is achieved, the Bremsstrahlung radiation generated would be mainly forward (regarding incidence direction). Due to the fact that electrons impact the surface of a spherical cap perpendicularly, the desired convergence effect should be produced on the forward-generated Bremsstrahlung radiation, according to its angular distribution at high energies. In this range of energy, most of the secondary photons produced by Bremsstrahlung radiation would also tend to be forward. The second step regards the beam convergence improvement by means of dedicated collimation and filter systems. Conceptual comparisons between conventional and convergent beam radiotherapy along with the corresponding dose profiles are depicted in figure 1 .

A simplified sketch and associated general overview are reported in figure 2 . The first stage starts with a standard electron beam from a linear accelerator (a), which is further split by a dedicated electron-dispersive sheet or electric/magnetic deflector (b). The next step consists of redirecting the electron beam with other electrostatic and/or magnetic elements (c), with the aim of attempting perpendicular impact on the surface of a thin curved anode (d). As a consequence of this process, $\mathrm{x}$-ray Bremsstrahlung is generated, which is mainly directed at the anode focus. Once photons emerge from the anode shell, they pass through a filter (e) and then through curved multiple-collimation systems (f). These multiple-collimation systems are made up of little conical holes (septa) directly pointing to the focus. Therefore, when photons produced by the Bremsstrahlung leave the generating device behind, they constitute an array of quasi-parallel micro beams that generate a single convergent beam when they are all considered together. Convergence might be applied either to the entire beam or limited to a specific conical shell.

The proposed design was developed to enable its adaptation either to a standard clinical linear accelerator or to a standalone CBRT device.

\subsection{Modeling dose distribution for analytical framework of a simple convergent beam}

Nowadays, teletherapy techniques are based on a wide range of modalities for producing a radiation beam. However, all the available technologies use conventional non-convergent beams. Dose concentration is achieved by combining multiple radiation fields or using beam modulation mechanisms.

When considering only geometry effects, i.e. neglecting attenuation in air, the convergent photon fluence in a vacuum can be simply assessed by considering inverse-square law (figure 3). Due to continuity requirements, the radiant energy flux at any h depth of the cone is constant at any transverse plane. Therefore:

$$
\Phi(h)=\Phi(0)=\Phi_{0}
$$

As the energy flow is equal to the energy fluence rate by intersecting the cone section, the above equation can be written as: 


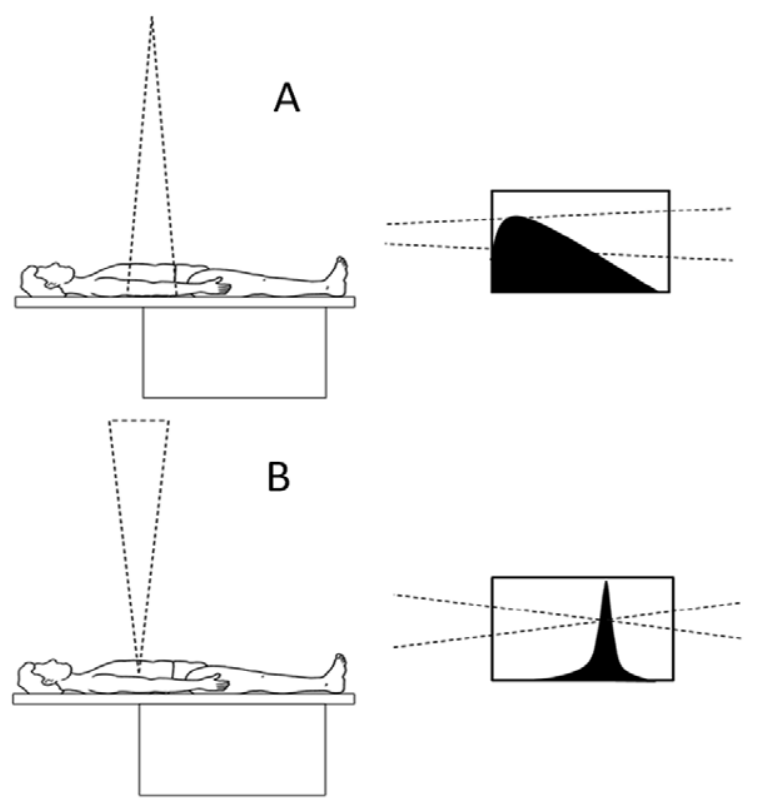

Figure 1. Conventional radiotherapy with divergent beam (A) and therapy proposed here (B), both with their respective depth dose profiles.

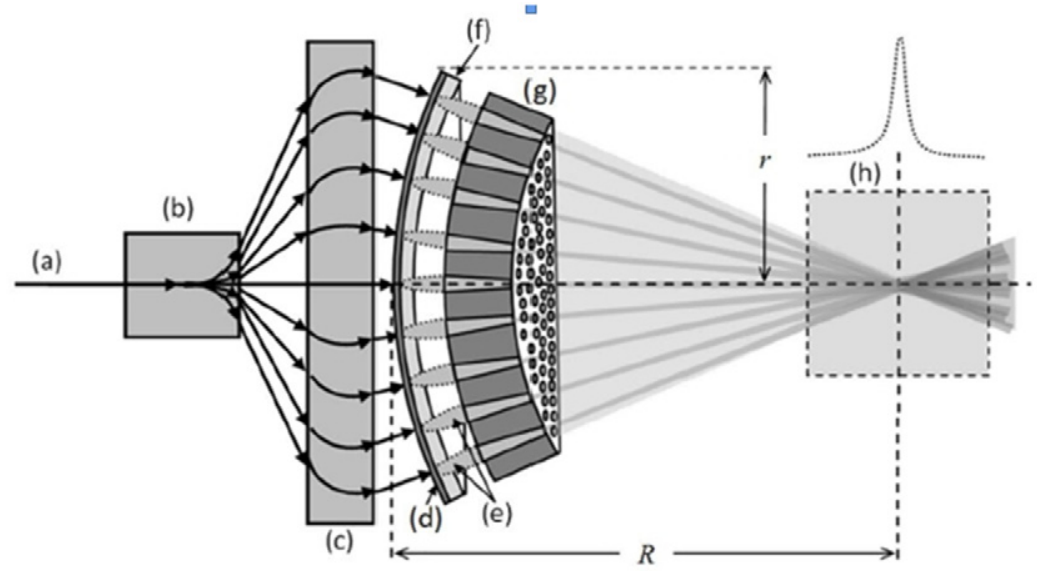

Figure 2. CBRT basic geometry: standard electron beam from a linear accelerator (a), dedicated electron-dispersive sheet or electric/magnetic deflector (b), other deflector electrostatic and/or magnetic elements (c), thin curved anode (d), filter (e), Bremsstrahlung cones emission (f), curved multiple-collimation systems (g) and phantom (h).

$$
\psi(h) \Delta(h)=\psi_{0} \Delta_{0}
$$

According to figure 3 the cone section at the input (0) is given by:

$$
\Delta_{0}=L_{0}{ }^{2} \Delta \Omega_{0}
$$

and the cone section to the $h$ depth is given by: 


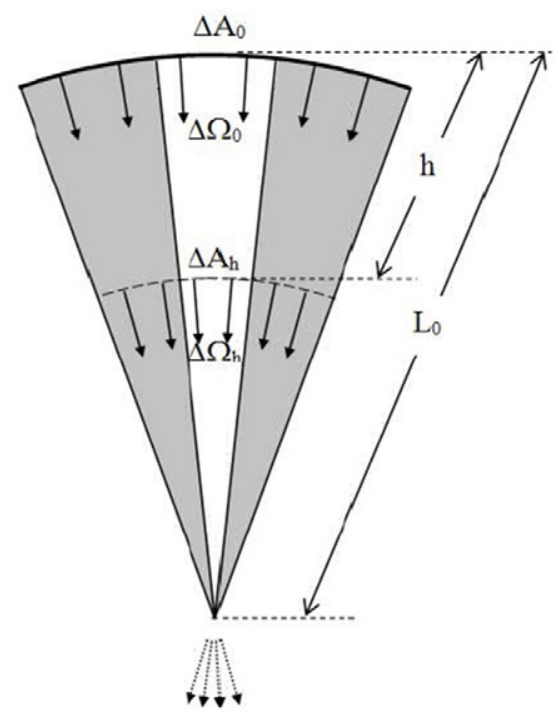

Figure 3. Diagram of cone fluence according to ideal convergent photon with attenuation.

$$
\Delta(h)=\left(L_{0}-h\right)^{2} \Delta \Omega_{h}
$$

Therefore, substituting in equation (1) and solving for the energy fluence rate at an $h$ depth inside the cone, the following is obtained:

$$
\psi(h)=\psi_{0} \frac{L_{0}^{2} \Delta \Omega_{0}}{\left(L_{0}-h\right)^{2} \Delta \Omega_{h}}
$$

According to figure $3, L_{\mathrm{o}}$ is the surface-isocenter distance and $\Delta \Omega_{0}$ and $\Delta \Omega_{\mathrm{h}}$ are the corresponding angular apertures at $L_{\mathrm{o}}$ and $h$ respectively.

The energy fluence rate calculated above describes only the geometrical dependence. When considering radiation flow within material media, radiation absorption and scattering need to be taken into account.

It is well known that scattering effects are difficult to accurately describe using simplified models. However, as a first approach, it might be acceptable to approximate the total energy fluence as the primary component, according to the standard attenuation law. Therefore, combining geometrical and physical effects for a hypothetical convergent x-ray beam and continuity conditions $\Delta \Omega_{0}=\Delta \Omega_{h}$, the energy fluence for an irradiation time equal to $t$ would be given by:

$$
\Psi(h)=\Psi_{0} \frac{L_{0}^{2}}{\left(L_{0}-h\right)^{2}} \mathrm{e}^{-\mu(E) \rho h}
$$

because $\Psi=\psi t$ and where $\mu(E)$ is the mass attenuation coefficient of the irradiated medium with mass density $\rho$, at the incident beam's energy $E$. In a first approximation, we can say that the resulting dose is directly proportional to the energy fluence. Therefore it will have the same form as that of equation (3), namely:

$$
D(h)=D_{0} \frac{L_{0}^{2}}{\left(L_{0}-h\right)^{2}} \mathrm{e}^{-\mu(E) \rho h}
$$




\subsection{Monte Carlo simulation (MC)}

In order to verify the proposed simplified analytical model, dedicated Monte Carlo simulations have been carried out. Specially adapted subroutines have been developed based on the PENELOPE v. 2008 main code. Simulation setup arrangements reproduce exactly both the geometrical and physical radiation transport properties considered for the analytical model, in order to consider converging Bremsstrahlung photons arising from a suitable emission shell and respective collimation devices.

\subsection{MC real case}

The implemented simulation mechanism allows the calculation of dosimetric and other physical properties, considering different user-defined configurations for the emission source, such as collimator aperture and anode size. Different incident energy values have been studied for the collision of the electron beam with the target. Therefore, the produced Bremsstrahlung X-ray beam will exhibit different spectral characteristics according to both the incident electron beam and the target's properties. The main characteristics of the proposed technique at different effective energies have been carefully studied considering 0.4 and $4 \mathrm{MeV}$. Monte Carlo simulations have been carried out for $10^{9}$ primary showers to ensure good enough uncertainty levels (a standard deviation of less than $0.1 \%$ ).

The approach selected to study the performance of the proposed method is built upon considering simplified situations as a starting point and then gradually moves to more complex real situations. Finally, a general overview and discussion with regard to the main characteristics of the proposed device, capable of generating a convergent photon beam and the electric and magnetic field necessary for CBRT geometry, will be described.

Next, MC simulations are performed using more realistic setups. An incident electron beam was transported in vacuum, whereas photons from the Bremsstrahlung yield and secondary particles were fully transported in the corresponding materials that include the high $\mathrm{Z}$ parts of the $\mathrm{x}$-ray production device, air and water. These simulations are performed for a thin target in the shape of a large, thin, spherical cap, with an radius $(15 \mathrm{~cm})$ and a curvature radius $\mathrm{R}(1 \mathrm{~m})$ and a cubed water phantom centered in the focal point of the cap. This way, the electrons perpendicularly impact upon various points of the cap (CBRT condition, convergent beam radiotherapy), aimed at the focal point. The $\mathrm{X}$ radiation (Bremsstrahlung) forwardly generated is responsible for the dose in the volume of the cubed phantom. All the interaction mechanisms of the $\mathrm{X}$ photons with the phantom are taken into consideration for different energies and cap thicknesses.

The emerging photon beam is emitted mainly convergent, despite the location where electrons reach the shell anode. The shielding structure allows for the required vacuum and protects the outside, thus avoiding external radiation emission. Finally, the emerging photon beam constitutes an almost convergent beam pointing to the focus geometrically determined by shell pieces, namely the anode, collimation grid and filtering accessories. It is not possible to ensure that all the photon fluence will arrive at the phantom, because of losses in the filtering and collimation systems. Therefore, it becomes crucial to characterize the angular distribution of the Bremsstrahlung yield. As is known, dependence on angular distribution and the kinetic energy of Bremsstrahlung production are intrinsically coupled so that it is necessary to investigate both of these parameters simultaneously affecting Bremsstrahlung yield. The key is that angular distribution probabilities become more and more closely concentrated around the forward direction for higher electron kinetic energies in the megavoltage range. Electrons carrying kinetic energy $T$ generate a photon of energy $E_{\mathrm{ph}}$ ranging from 0 to $T$. Thus, 
the atomic differential cross section $\left(\mathrm{d} \sigma_{\mathrm{Br}}\right)$ is the differential in the energy loss $E_{\mathrm{ph}}$ as well as the projectile final direction and emitted photon direction. According to the Bethe-Heitler Bremsstrahlung model, radiation yield produced by electrons in the field of an atom of atomic number $Z$ with screening radius $R_{S}$ is expressed as:

$$
\frac{\mathrm{d} \sigma_{\mathrm{Br}}}{\mathrm{d} T}=Z(Z+\eta) \frac{\alpha}{T} r_{0}^{2}\left[\varepsilon^{2} F(b)+\frac{4}{3}(1-\varepsilon G(b))\right]
$$

where $\alpha$ is the hyperfine structure constant, and the parameters $\varepsilon$ and $b$ are respectively defined by $\varepsilon=E_{\mathrm{ph}} /\left(\gamma m_{e} c 2\right)$ and $b=R m_{e} c \varepsilon /(2 \gamma h(1-\varepsilon))$, both depending on relativistic parameter $\gamma=$ $\left(1+(v / c)^{2}\right)^{-1 / 2} ; F$ and $G$ are combinations of functions depending upon the order of expansion used to model the Bremsstrahlung yield. Considering cylindrical symmetry for isotropic media, the Bremsstrahlung photon direction is completely defined by the polar angle $\theta$, so that $d \sigma_{B r}$ can be expressed as:

$$
\frac{\mathrm{d}^{2} \sigma_{\mathrm{Br}}}{\mathrm{d} E_{\mathrm{ph}} \mathrm{d}(\cos (\theta))}=\frac{Z^{2}}{\beta^{2}} \frac{1}{E_{\mathrm{ph}}} A(Z, T, \kappa) B(Z, T, \kappa, \cos (\theta))
$$

In the above equation $(6), A(Z, T, \kappa)$ is a parameter dependent on the target material and $\kappa=E_{\mathrm{ph}} / T$. The shape function $B(Z, E, \kappa, \cos (\theta))$ is the partial differential function of $\cos (\theta)$ providing angular distributions of the Bremsstrahlung yield according to Sommerfeld's theory:

$$
B(Z, T, \kappa, \cos (\theta))=\frac{\sigma_{I}\left(1-\cos ^{2}(\theta)\right)+\sigma_{I I}\left(1+\cos ^{2}(\theta)\right)}{\sigma_{I}\left(1-\beta \cos ^{2}(\theta)\right)}
$$

$\sigma_{I}$ and $\sigma_{I I}$ are parameters that do not depend on the polar angle $\theta$. The simplified expression in equation (7) assesses the overall global trends of the partial-wave shape functions. Moreover, these parameters can be obtained by suitable fittings extracted from Sommerfeld's model.

In order to carry out MC simulations, geometrical configuration and experimental setups were implemented according to figure 2. However, for the simplified preliminary approach, electron beam deflection was not introduced. Thus, the starting point was an electron beam impinging upon the shell anode normally. Particle cascades (showers) were simulated up to primary and secondary particle absorption; hence the performed MC simulations calculated the in-phantom absorbed dose due to the Bremsstrahlung photons produced by the electron beam colliding with the shell anode.

$\sigma_{I}$ and $\sigma_{I I}$ are parameters that do not depend on the polar angle $\theta$. The simplified expression in equation (3) assesses the overall global trends of the partial-wave shape functions. Moreover, these parameters can be obtained by suitable fittings extracted from Sommerfeld's model.

\subsection{Field necessary for electron trajectory in CBRT geometry}

An S-shaped path is followed by the electrons in the CBRT geometry, shown in figure 2, before impacting on the anode. The two radii of curvatures $\rho_{A}$ and $\rho_{C}$ are illustrated below in figure 4 .

The electric and magnetic fields were investigated in order to assess the suitable mechanism for electron beam deflections as required for implementing CBRT devices. To this end, the relativistic electromagnetism formalism was used for the study of a wide range of electron energy ( 0.1 up to $20 \mathrm{MeV}$ ) and desired curvature radii.

The control parameters associated with the electric and magnetic fields necessary to bend electron beams reveal that this is feasible. However, magnetic fields exhibit values easier to achieve than electrostatic ones. These results were obtained by a relativistic equation for an electron move: 


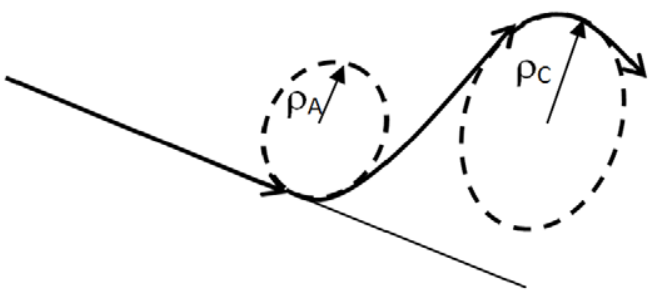

Figure 4. Electron path in solid line with arrows in CBRT geometry, opening curvature radius $\rho_{\mathrm{A}}$, closing curvature radius $\rho_{\mathrm{C}}$.

$$
\frac{\mathrm{d} \vec{p}}{\mathrm{~d} t}=\gamma m_{0} \frac{\mathrm{d} \vec{v}}{\mathrm{~d} t}+m_{0} \vec{v} \gamma^{3} \frac{\beta}{c} \frac{\mathrm{d} v}{\mathrm{~d} t}
$$

Where $\gamma$ is the relativistic factor, $\vec{v}$ is the electron velocity, $\beta$ is the velocity factor with respect to light speed $c$ and $m_{0}$ is the electron mass. For the perpendicular force condition equation (4) is reduced to:

$$
\frac{\mathrm{d} p}{\mathrm{~d} t}=\gamma m_{0} \frac{v^{2}}{\rho}
$$

where $\rho$ is the turn ratio of the electron trajectory. If the perpendicular force is generated by an electric field, the intensity of the electric field $E$ is in this case related to the turning radius and the kinetic energy through:

$$
E=\gamma m_{0} \frac{v^{2}}{\mathrm{e} \rho}=\gamma \frac{m_{0} c^{2}}{\mathrm{e} \rho}\left[1-\frac{1}{(1+T)^{2}}\right]
$$

where $T$ is the electron kinetic energy. In this case, the intensity of the magnetic field $B$ is related to the turning radius and kinetic energy through:

$$
B=\gamma m_{0} \frac{v}{\mathrm{e} \rho}=\gamma \frac{m_{0} c}{\mathrm{e} \rho} \sqrt{\left[1-\frac{1}{(1+T)^{2}}\right]}
$$

The magnitudes of the electric and magnetic fields necessary for the CBRT geometry proposed here were calculated from equations (7) and (8) respectively, for energies of 0.1 to 20 $\mathrm{MeV}$ and three different radii of curvature, 10,20 , and $30 \mathrm{~cm}$.

\section{Results and discussion}

\subsection{Dose profile comparisons between convergent beams}

Figure 5 shows the dose profile result achieved using equation (6) for an ideal convergent beam with $(L o-h)_{\text {Min }}=0,2 \mathrm{~cm}$, focus size $0,8 \mathrm{~m}$ approximately. This on-axis profile corresponds to the calculation for the central axis, integrated in the voxel $\left(1 \times 1 \times 1 \mathrm{~mm}^{3}\right)$ centered in the $z$-axis. Unlike in conventional techniques, there is an unexpected dose profile focused on the isocenter or focal point of the system. This dose profile is similar in shape to a peak and has virtually zero dose at the entrance.

Figure 5 shows a typical in-depth dose profile for a hypothetical convergent beam.

Figure 6 shows in-depth dose profiles for a hypothetical convergent beam, corresponding to different attenuation coefficients. From a general standpoint, the in-depth focus position 


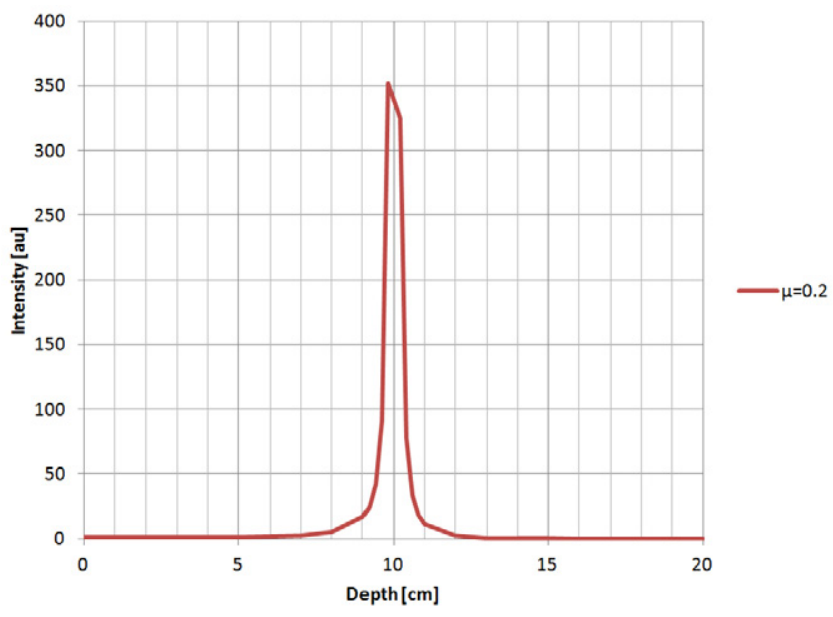

Figure 5. In-depth dose profiles evaluating the case $\mu=0.2 \mathrm{~cm}^{-1}$ and isocenter to $10 \mathrm{~cm}$ from surface.

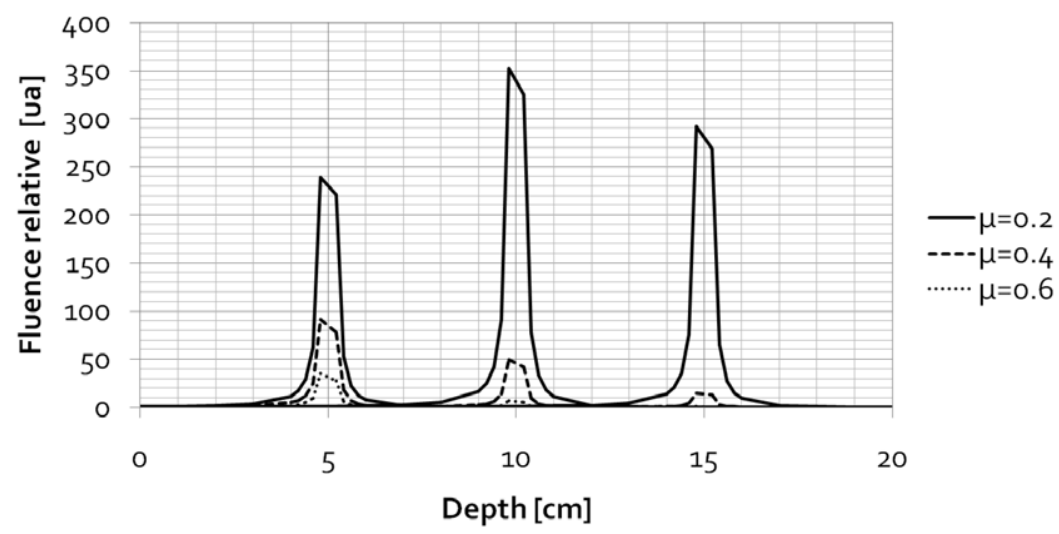

Figure 6. In-depth dose profiles for different combinations of attenuation coefficient $\mu(E)$, expressed in $\mathrm{cm}^{-1}$, corresponding to focus positions 5,10 and $15 \mathrm{~cm}$ in depth.

depends upon the physical parameters and irradiation setup. Generalization to arbitrary spectra is straightforward introducing the corresponding fluence. Therefore when considering typical cases of teletherapy applications, i.e. those involving megavoltage x-ray beams and biological tissues, it is observable that the focus of the radiation delivered exhibits weak dependence on the energy beam. The specific design and irradiation setup configuration is decisive.

It is noticeable that to keep the maximum intensity at different focus peak depths, it is slightly lower than the intensity at the entrance for the case of $\mu=0.6 \mathrm{~cm}^{-1}$. Even in this situation, the maximum remaining dose is considerably higher than the input intensity, and focus might be considered as a confirmation of the already mentioned quasi-independence with respect to photon energy, being strongly dependent on the device design and irradiation setup.

Figure 7 reports comparisons between the analytical model and Monte Carlo simulated depth dose curves. It should be noted that the Monte Carlo technique takes into account scattering effects, while the proposed simplified analytical model considers only the primary radiation component. However, it should be highlighted that there is a remarkable agreement 


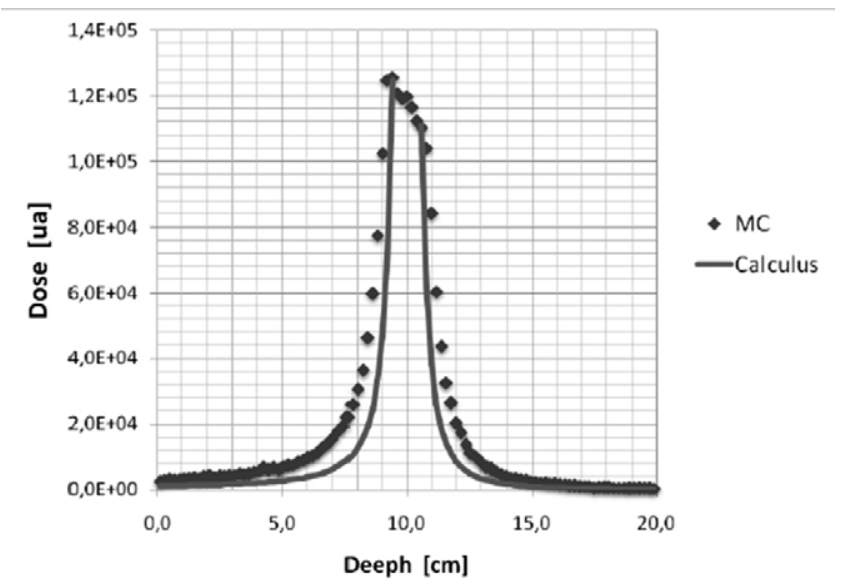

Figure 7. Comparison between analytical model (solid line) and Monte Carlo simulations (diamond points) corresponding to a $2 \mathrm{~mm}$ size spot, $10 \mathrm{~cm}$ depth focus, irradiating a water phantom with $4 \mathrm{MeV}$ energy of photons directed at the focus point.

between the obtained results for on-axis dose distributions, excluding the region around the focus, where the analytical approach predicts significantly higher values. These differences might be attributable to in-depth primary beam degradation effects that are not considered in the simplified analytical model.

\subsection{MC simulation for real convergent beam}

In general, the $z$ in-depth focus position depends only on the geometry. Therefore, within the framework of radiotherapy applications, i.e. the typical energy range and irradiated organic materials, it can be concluded and stated that focus position is almost independent of beam energy. The performance of standard x-ray generator devices might be characterized by their dosimetric capability since virtually all other physical properties and design parameters have been largely studied. However, the properties of the prototype proposed for convergent X-ray generation require further research in order to establish, at least preliminarily, its feasibility, its global and dosimetric performance, and its corresponding reliability. Bremsstrahlung was studied as a first step, varying the design parameters of the potential anode prototype, with specific characteristics and operating under different configurations. Figure 8 shows the effect of the anode characteristics on Bremsstrahlung performance, corresponding to a specially designed poly-collimation grid, sketched in figure 9.

According to the achieved high dose concentration, the obtained preliminary results suggest the potentiality of such an incident beam for teletherapy. In this regard, the design and development of suitable devices capable of delivering a convergent beam is extremely relevant, in order to assess novel and feasible techniques for radiation therapy by means of a single external photon beam.

The CBRT technique proposal might offer several relevant and valuable advantages related to the high-dose level at the focal zone (spot) while keeping surrounding tissues at significantly lower dose values. This might reduce the required number of treatment sessions as well as increase the rate of treated patients. In addition, due to the high performance and functionality of the proposed method, even for the orthovoltage range, other benefits may arise from cost reduction on radiologic protection. 

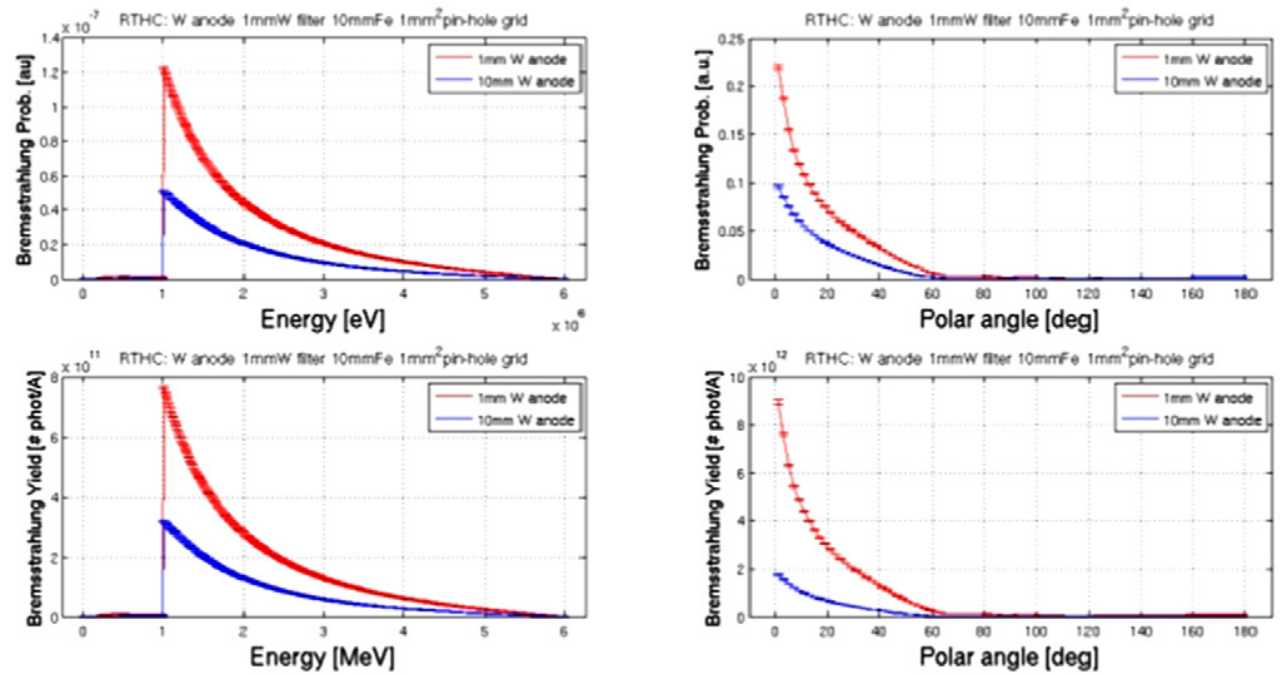

Figure 8. Effect of anode's thickness on Bremsstrahlung production with a $6 \mathrm{MeV}$ electron beam. Top: energy and angular distribution of Bremsstrahlung production probability. Bottom: Bremsstrahlung production per current unit (Ampere) of the incident electron beam. The simulation of CBRT is referred to as 'RTHC Bremsstrahlung', as indicated in the figure.

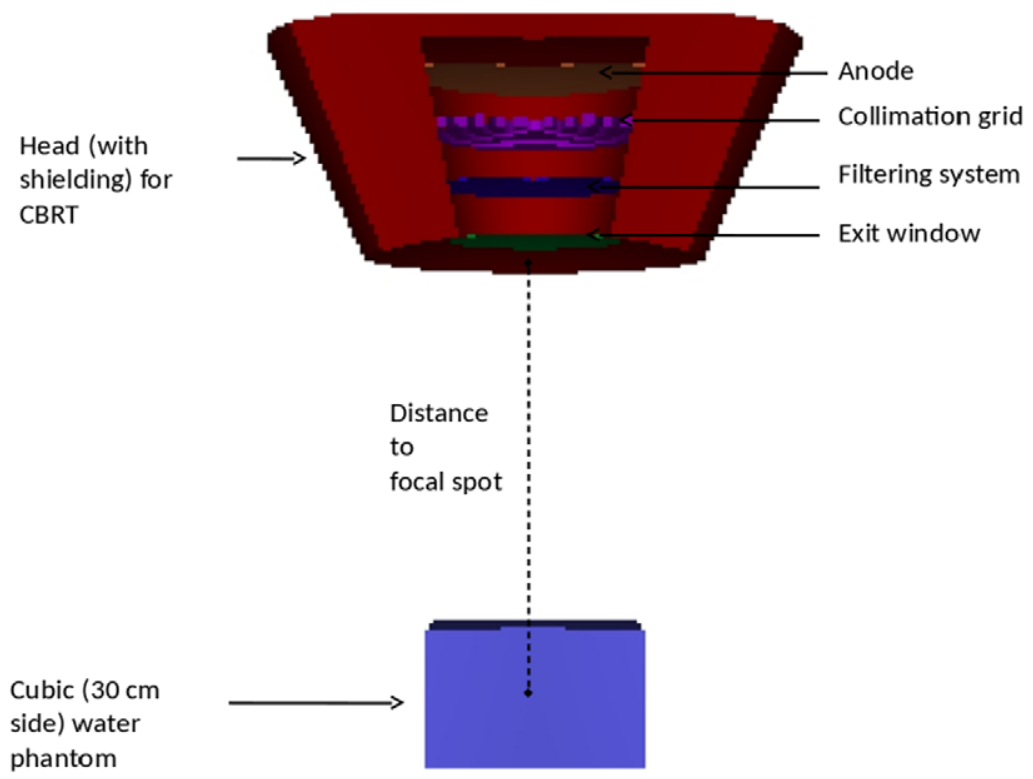

Figure 9. Setup for CBRT convergent beam device considered in the Monte Carlo simulation indicating: the spherical shell anode, collimation grid and filtering.

It was observed that combining the collimation and cone angle of the convergent $\mathrm{x}$-ray beam allows control of dose point size as well as dose values within regions around the focus. As the collimation solid aperture angle increases and the cone opening decreases, the focal 

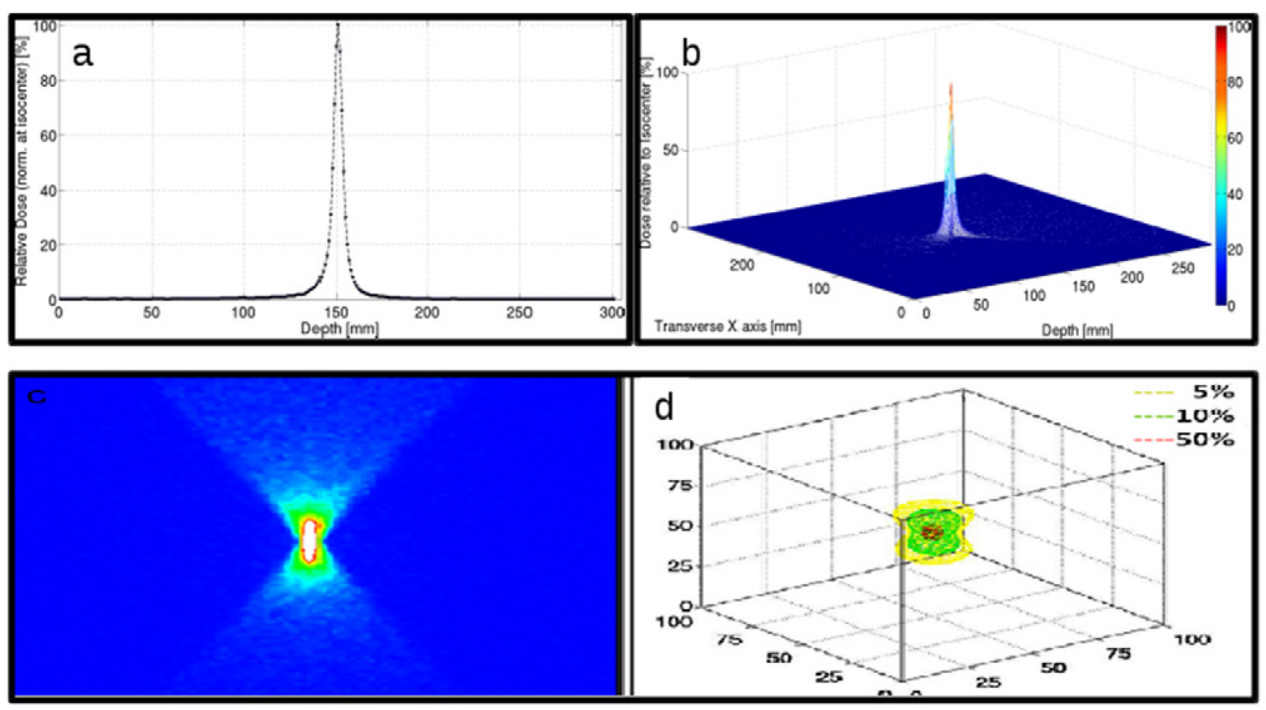

Figure 10. Dose distribution inside cubic a water phantom obtained with $400 \mathrm{keV}$ convergent photon beam. Analytical in-depth dose profile (a) and 3D (b) representations along dose distribution in the central plane (c) and volumes of isodose 3D representation (d) obtained from MC simulations.

zone is extended and acquires lesser dose values. The smaller the collimator's solid aperture angle and the greater the opening of the cone of convergence, the smaller the focal zone will be, but the greater the intensity.

In summary, as the dimensions of the focal zone (spot) decrease, the dose values increase. It is conceptually like a Dirac delta function. However, in practice the maximum value will always be finite due to the non-null angular aperture of the HCX poly-collimator.

When the results obtained with the analytical model are compared with those found using Monte Carlo simulation, it is observable that the latter also reveals the presence of a peak in the system focus that is essentially equal to that in the analytical model. However, there are differences with regard to the peak's intensity and width since the theoretical peak might practically have a punctual focus with infinite intensity, as opposed to the peak achieved by Monte Carlo simulation, which has a certain width due to dispersion, which is not considered in the theoretical approach.

For smaller convergence angles, under 20 degrees of the cone, a clear shift of the focus position is generated. In-depth dose profiles in the water phantom show good concordance between the theoretical predictions and the results obtained by Monte Carlo simulations from photons emerging from a source. This is in accordance with the expected outcomes, providing a dose profile that in ideal terms will have a maximum in the focus and is similar to a Dirac delta in shape, which is independent of the photon energy but whose intensity is highly dependent on the material, energy and convergence angle.

For typical beams (photons of $1.25 \mathrm{MeV}$ and water), ideal values of 50 times the dose in the focus with respect to the dose at the entrance of the beam phantom will be obtained. It must be taken into account that a complete $\mathrm{MC}$ simulation generates a slightly wider and less intense beam than an analytic approach.

The results in figure 10 show the dose profile (a), 3D dose profile (b), 2D dose distribution (c) and 3D dose distribution (d) achieved by $\mathrm{MC}$ simulation for a $400 \mathrm{keV}$ convergent photon 
Table 1. Electric field $E$ values and magnetic field values $B$ for various electron kinetic energies for three turning radii $(10,20$ and $30 \mathrm{~cm})$ of the curved trajectories of the electrons.

\begin{tabular}{|c|c|c|c|c|c|c|}
\hline $\begin{array}{l}\text { Electron } \\
\text { energy }\end{array}$ & $E$ & $B$ & $E$ & $B$ & $E$ & $B$ \\
\hline $\mathrm{T}$ & \multicolumn{2}{|c|}{ Turn radius $10 \mathrm{~cm}$} & \multicolumn{2}{|c|}{ Turn radius $20 \mathrm{~cm}$} & \multicolumn{2}{|c|}{ Turn radius $30 \mathrm{~cm}$} \\
\hline $\mathrm{MeV}$ & $\mathrm{KV} / \mathrm{cm}$ & Tesla & $\mathrm{KV} / \mathrm{cm}$ & Tesla & $\mathrm{KV} / \mathrm{cm}$ & Tesla \\
\hline 0.1 & 18.5 & 0.010 & 9.2 & 0.006 & 6.2 & 0.004 \\
\hline 0.3 & 49.2 & 0.020 & 24.6 & 0.011 & 16.4 & 0.007 \\
\hline 0.5 & 75.7 & 0.03 & 37.9 & 0.015 & 25.2 & 0.010 \\
\hline 1 & 134.7 & 0.050 & 67.3 & 0.024 & 44.9 & 0.016 \\
\hline 3 & 345.8 & 0.120 & 172.9 & 0.058 & 115.3 & 0.039 \\
\hline 6 & 651.1 & 0.220 & 325.6 & 0.109 & 217.0 & 0.073 \\
\hline 10 & 1055.2 & 0.350 & 527.6 & 0.176 & 351.7 & 0.117 \\
\hline 20 & 2062.6 & 0.600 & 1031.3 & 0.344 & 687.5 & 0.229 \\
\hline
\end{tabular}

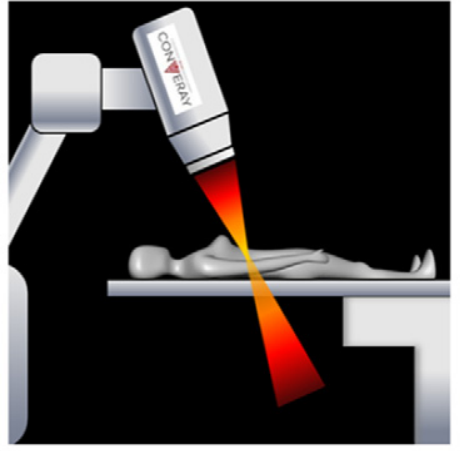

(a)

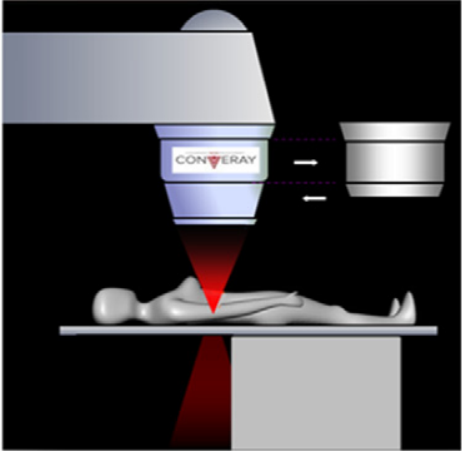

(b)

Figure 11. Sketch for the design of CBRT devices: standalone robotic apparatus (a) and Converay ${ }^{\circledR}$ adapted to conventional LINAC.

beam irradiating a water phantom. As expected, notice that high doses concentrate close to the isocenter; whereas surrounding regions are kept at very low dose levels as also happens in the entrance.

\subsection{Field and electron trajectory for CBRT}

Table 1 reports the values obtained for the electric and magnetic fields in the case of 3 different curvature radii that are required for attaining satisfactory electron beam deflection when considering electron beam energies in the range of $0.1-20 \mathrm{MeV}$.

The control parameters associated with the electric and magnetic fields necessary to bend the electron beams reveal that this is feasible. However, the magnetic fields exhibit values that are easier to achieve than the electrostatic ones.

Considering typical clinical situations (electron beam energy of around $6 \mathrm{MeV}$ might be representative), it was found that electron beam deflection does not seem to represent technical 
difficulties to be attained by means of conventional magnetic fields. In fact, $0.22 \mathrm{~T}$ would be enough. On the other hand, electric fields might require extremely large intensities, thus avoiding its implementation for electron beam energies greater than $1.0 \mathrm{MeV}$ approximately.

Finally, a simplified sketch of CBRT implementation is reported in figure 11 .

After the investigation of the main characteristics of the proposed CBRT technique, it may be pointed out that the performance of such a prototype could represent a valid alternative to perform a variety of practical applications. The irradiation of small targets surrounded by critical structures appears as a part of the scope that may be considered. Then, the capability of CBRT devices may constitute significant advantage for treatments requiring high dose concentrations to irradiated relatively small targets surrounded by healthy tissues, like radiosurgery. In addition, CBRT would not be limited to the treatment of small targets, because larger volumes might be treated by implementing dose-painting techniques by means of mechanically moving the head of the prototype, changing the accelerating voltage of the electron beam, or varying the parameters in the focusing electromagnetic device, among other available options to manipulate and control dose delivery.

\section{Conclusions}

Besides preliminarily showing how to achieve a convergent beam, the CBRT technique for developing a prototype capable of producing convergent beams of photons for teletherapy application was studied. The simplified analytical model was successfully tested by comparison with the performed Monte Carlo simulations of fully stochastic radiation transport. It was preliminarily shown as well that the proposed method is capable of assessing opportune and appropriate $3 \mathrm{D}$ dose distributions.

Although the analytical description of primary radiation propagation was significantly simplified, it should be remarked that the obtained results are in good agreement with complete radiation transport and interaction by Monte Carlo simulations.

With regard to the proposed design and associated prototype for convergent beam production, it should be mentioned that the obtained results show the feasibility of controlling electron trajectories through electric and/or magnetic fields by splitting the beam before its impact on the spherical anode shell in such a way that virtually all the photons emitted by the Bremsstrahlung move forward directly toward the focus. The high atomic number of the spherical anode shell makes it possible to obtain convergent beams by means of the conical apertures of the shell, between 40 and 60 degrees.

Bremsstrahlung 'production' is generated by the impact on the spherical shell. It was verified that its emission is mainly directed forward, pointing to the focus. Before leaving the CBRT device, $\mathrm{x}$-rays pass through specific grid systems for filtering and collimation, which show potential to improve beam convergence for the further delivery of highly concentrated dose distributions around the focal zone, while the surrounding areas are affected with very low doses.

The lenses' electrostatic fields necessary to control the electron trajectories are difficult to achieve for megavoltage electron currents. Therefore, it is more convenient to use magnetic fields for electron energies higher than approximately $1 \mathrm{MeV}$, because this might be achieved using a field intensity lower than a tesla. Thus, it is possible to significantly reduce the required electron current.

Therefore, within the low-energy range used in external radiation therapy, up to $6 \mathrm{MeV}$, it is feasible to construct a magnetic and/or electrical device for the deflection of the electron trajectories required for the CBRT geometry with the operational values of the current 
and voltage available. Additionally and according to the presented results, it is necessary to highlight that the proposed technique might also be adapted and successfully implemented at low-energy (orthovoltage) irradiation facilities.

\section{Acknowledgments}

The authors wish to thank the Universidad de La Frontera, CONICET, Universidad Nacional de Córdoba, and CORFO-Chile, (Project 12IDL1-13124-RTHC (CBRT)) for supporting the patent applications and this research.

\section{References}

Anderson E S, Muren L P, Sørensen T S, Noe K O, Thor M, Peterson J B, Høyer M, Bentzen L and Tanderup K et al 2012 Bladder dose accumulation based on a biomechanical deformable image registration algorithm in volumetric modulated arc therapy for prostate cancer Phys. Med. Biol. 57 7089-100

Andrews D W, Bednarz G, Evans J J and Downes B 2006 A review of 3 current radiosurgery systems Surgical Neurology 66 559-64

Bassalow R and Rodebaugh R 2006 SU-FF-T-227: evaluation of six dosimetric indices for cyber knife stereotactic radiosurgery treatment planning Med. Phys. 332100

Bohoslavsky R, Witte M G, Janssen T M and van Herk M 2013 Probabilistic objective functions for margin-less IMRT planning Phys. Med. Biol. 58 3563-80

Cui Y et al 2013 Implementation of remote 3-dimensional image guided radiation therapy quality assurance for radiation therapy oncology group clinical trials Int. J. Radiat. Oncol. Biol. Phys. 85 271-7

Das $\mathrm{S}$ et al 2003 Beam orientation selection for intensity-modulated radiation therapy based on target equivalent uniform dose maximization Int. J. Radiat. Oncol. Biol. Phys. $55215-24$

Figueroa R G and Valente M 2013 Generator apparatus of convergent beams of electron beams and X-rays Patent Applied, Chile; 898 -2011 Argentina, (2012), PCT; (2013) (2013)

Freeman T and Hd V 2013 F T Elekta unveils Versa HD

Jaffray D, Kupelian P, Djemil T and Macklis R M 2007 Review of image-guided radiation therapy Expert Rev. Anticancer Ther. 7 89-103

Kestin L L et al 2000 Intensity modulation to improve dose uniformity with tangential breast radiotherapy: initial clinical experience Int. J. Radiat. Oncol. Biol. Phys. 48 1559-68

Mackie T R et al 1993 Tomotherapy: a new concept for the delivery of dynamic conformal radiotherapy Med. Phys. 20 1709-19

Maxim P G, Fahimian B P and Xing L 2011 Image guidance on the truebeam STx: evaluation of CBCT imaging dose and quality Radiat. Oncol. 81 S849-50

Meltsner S and DeWerd L 2008 TU-FF-A2-05: 3D dose distribution of the elekta gamma knife Med. Phys. 352921

Nie K et al 2012 SU-D-211-04: sector intensity modulated (SIM) gamma knife stereotactic radiosurgery Med. Phys. 393610

Otto K 2008 Volumetric modulated arc therapy: IMRT in a single gantry arc Med. Phys. 35 310-7

Phillips M H, Singer K M and Hounsell A R 1999 A macropencil beam model: clinical implementation for conformal and intensity modulated radiation therapy Phys. Med. Biol. 44 1067-88

Spirou S V and Chui C S 1994 Generation of arbitrary intensity profiles by dynamic jaws or multileaf collimators Med. Phys. 21 1031-41

Szeifert G T et al 2008 The gyroknife digital cobalt-60 stereotactic radiotherapy and radiosurgery system Hungarian Med. J. 2 55-62

Verellen D et al 2007 Innovations in image-guided radiotherapy Nat. Rev. Cancer 7 949-60

Webb S 2003 The physical basis of IMRT and inverse planning Br. J. Radiol. 76 678-89

Yao-zhen L, Xian-Shi H and Zhen-Kai Y E 2013 Clinical observation of treatment of 11 malignant tumor patients with cancer embolism in deep vein of lower extremities by the radiation therapy of gyro rotary 60 cobalt radiation treatment system Chin. J. New Clin. Med. 25

Yu C X 1995 Intensity-modulated arc therapy with dynamic multileaf collimation: an alternative to tomotherapy Phys. Med. Biol. 40 1435-49 